\title{
OPTIMAL CONTROL APPLIED TO HEPATITIS C THERAPY CONSIDERING IMMUNE SYSTEM
}

\author{
ALEJANDRO PEREGRINO ${ }^{1}$, LOURDES ESTEVA ${ }^{2}$ \\ and GAMALIEL BLÉ ${ }^{1}$
}

${ }^{1} \mathrm{DACB}, \mathrm{UJAT}, \mathrm{Km} 1$

Carretera Cunduacán-Jalpa de Méndez

C. P. 86690 Cunduacán Tabasco

México

e-mail: alejandro.peregrino@ujat.mx

gble@ujat.mx

2Facultad de Ciencias

UNAM

D. F. C. P. 04510

México

e-mail: lesteva@ciencias.unam.mx

\begin{abstract}
We present a mathematical model for the dynamics of hepatitis $\mathrm{C}(\mathrm{HCV})$. We included in the model treatment with combination therapy of interferon and ribavirin. In order to provide a treatment regimen that minimizes both economic cost and side effects, we propose and solve numerically an optimal control problem considering the cost of treatment and its side effects in the patient. The optimal control is obtained by applying the Pontryagin maximum principle.
\end{abstract}

2010 Mathematics Subject Classification: 49J15, 92B05, 49K35.

Keywords and phrases: hepatitis C, optimal control, Pontryagin maximum principle.

Received November 30, 2017; Revised February 8, 2018.

(C) 2018 Scientific Advances Publishers 


\section{Introduction}

Hepatitis $\mathrm{C}$ virus infection (HCV) is a public health problem with strong clinical and economic implications. The HCV may progress to a chronic acute infection, liver cirrhosis (20\% to $30 \%$ ) or liver cancer (with a much smaller percentage), both of deadly consequences [9]. According to WHO (2000), around 130 to 170 million people worldwide are carriers of HCV [18].

The HCV infects liver cells, causing that they no longer work properly. The most frequent mechanisms of transmission of HCV are blood transfusions, intravenous drug use, hemodialysis, tattoos, risky sexual behaviours, vertical transmission from mother to child and transplants, among others. The incubation period of HCV is around 50 days and the evolution time is 10 to 20 years (the time of onset of cirrhosis is 21.2 years, while the liver cancer is about 29 years).

The infection becomes chronic in most cases, and the high mutation rate of the virus has prevented the development of an effective vaccine. For this reason the phenomenon of re-infection occurs [17].

Currently treatments approved by the Food Drug Administration (FDA) for chronic hepatitis $\mathrm{C}$ involve the application of interferon $\alpha-2 \alpha$ or $\alpha-2 b$ (INF) pegylated, more ribavirin. The interferons are a family of intracellular proteins and their main function is to block viral replication. The ribavirin is a synthetic guanosine nucleoside serving as antiviral.

There are treatments to assess liver damage, one of them is liver biopsy, which for years has been the best tool for diagnosing the progression of liver disease, although now its use has been reduce because there are various risk factors and complications.

The determination of viral load, resource fairly new, allows to know the intensity of infection. Therefore, the central goal of treatment is to eliminate or at least to reduce viral load. 
In order to understand the dynamics of $\mathrm{HCV}$, in particular, to determine the effectiveness of therapy with interferon and ribavirin, mathematical models have been used to explain the changes on viral load patterns observed in patients infected with HCV. Neumann et al. [15] describe the dynamics of HCV treated with interferon- $\alpha$ using a mathematical model based on three compartments: healthy liver cells, infected hepatocytes, and viral load. Dahari et al. [7] improved Neumann model taking into account the homeostatic mechanisms for the liver. Their model successfully explains the triphasic decline of virus load, as well as therapeutic failures.

In the same line of study of Neumann model, Dixit et al. [6] take into account the action of interferon with ribavirin. According to [3], the ribavirin is incorporated into RNA to increase the mutation frequency and reducing the specific infectivity of new virions, resulting in two populations: infectious and noninfectious virions. Their model suggests that ribavirin plays a minor role in the first phase of the reduction of viral load, however, when the efficacy of interferon is small, efficacy of ribavirin remarkably improved, giving support to the clinical data reported in [19]. Banerjee et al. [2] consider that the proliferation of hepatocytes occurs logistically and they do not distinguish between infected and uninfected hepatocytes.

Control theory has been used in a large number of application including biological and economical problems [12]. In the case of HCV, Chakrabarty and Joshi [5] motivated by [6, 15] consider a model for HCV dynamics under combination therapy of interferon and ribavirin. a functional objective is formulated in order to minimize the viral load, as well as the drug side-effects. The optimal system is solved numerically to determine optimal drugs efficacies. Chakrabarty [4] extended the results in [5] by considering a clinically validated functional form of the interferon efficacy, and hence they determined the optimal efficacy of ribavirin. Martin et al. [13] in a recent paper examine a three 
compartment model for $\mathrm{HCV}$, involving the susceptible, chronically infected and treated injection drug users (IDUs). They determine an optimal treatment programme over a 10 year period taking into account several biomedical and economical objectives. By last, Ntaganda et al. [14] analyze the role of drugs, and how they play a crucial role in controlling HCV diseases through a bicompartmental model such that the controls are those drugs.

In this work, we want to find the minimal effort necessary to reduce viral load considering the cost of the treatment, as well as the cost of the secondary effects derived from the medication. For this end, we take the model formulated in [1] as a base to apply the optimal control theory.

The paper is organized as follows. Section 2 presents the model equations. In Sections 3 and 4, treatment is included and the optimal control problem is formulated. Also, existence and uniqueness of the control problem are proved. The numerical results are shown in Section 5 , and finally conclusion is presented in Section 6 .

\section{The Mathematical Model}

The mathematical model for the dynamics of HCV proposed and studied in Avendaño et al. [1], is given by the following system of ordinary differential equations:

$$
\begin{aligned}
& \dot{H}_{s}=\beta_{s}-k H_{s} V-\mu_{s} H_{s}, \\
& \dot{H}_{i}=k H_{s} V-\delta H_{i} T-\mu_{i} H_{i}, \\
& \dot{V}=p H_{i}-\mu_{v} V, \\
& \dot{T}=\beta_{T} V\left(1-\frac{T}{T_{\max }}\right)-\mu_{T} T,
\end{aligned}
$$

for which the system variables and assumptions are as follows: 
- $H_{s}(t)$ is the population of healthy hepatocytes in the liver at time $t$ $\left(\right.$ cell $\left./ \mathrm{mm}^{3}\right)$. These are generated at a constant rate $\beta_{s}$ and die at a per capita $\mu_{s}$.

- $H_{i}(t)$ is the population of infected hepatocytes in the liver at time $t$ $\left(\right.$ cell $\left./ \mathrm{mm}^{3}\right)$. Healthy hepatocytes $H_{s}$ become infected $H_{i}$, with a rate proportional to the product of the number of healthy hepatocytes $H_{s}$ by viral load $V$, with proportionality constant $k$.

- $V(t)$ is the viral load at time $t\left(\frac{U I}{\mu L}\right)$. VHC virions are produced within an infected hepatocyte $H_{i}$, at a per capita $p$ virions per hepatocyte infected per day, and die with a death rate $\mu_{v}$. The infected hepatocytes $H_{i}$ die from HCV replication at a per capita $\mu_{i}>\mu_{s}$.

- $T(t)$ is the population of $T$ killer cells (cell type CD8+) at time $t$ $\left(\right.$ cell $\left./ \mathrm{mm}^{3}\right)$. The $T$ killer cell destroy infected hepatocytes $H_{i}$ at a rate proportional to the product of the number of infected hepatocytes $H_{i}$ times the number of $T$ killer cells, with proportionality constant $\delta$.

- Under the presence of HCV, the $T$ killer cells grow proportionally to the viral load $V$ with a saturation rate $\beta_{T}\left(1-T / T_{\max }\right)$, where $\beta_{T}$ is the rate of reproduction of $T$ killer cells, and $T_{\max }$ is the maximum $T$ killer cells in the body. Furthermore, these cells die at a per capita $\mu_{T}$.

We define the set of biological interest $\Omega$ as

$$
\Omega=\left\{\left(H_{s}, H_{i}, V, T\right) \in \mathbf{R}_{+}^{4} \backslash H_{s}+H_{i} \leq H_{M}, V \leq V_{M}, T \leq T_{M}\right\},
$$

where $H_{M}=\frac{\beta_{s}}{\mu_{s}}$ is the maximum population of healthy hepatocytes in the liver of a healthy individual, $V_{M}=\frac{p H_{M}}{\mu_{V}}$ the maximum viral load that a person can withstand, and $T_{M}=\left(\frac{\beta_{T}}{\mu_{T}^{*}}\right) V_{M}$ with $\mu_{T}^{*}=\mu_{T}+\left(\frac{\beta_{T}}{T_{\max }}\right) V_{M}$. 
The following results are given in [1].

Lemma 1. If $\mu_{i} \geq \mu_{s}$, then $\Omega$ is positively invariant for system (1).

Theorem 2. Let $R_{0}=\frac{k \beta_{s} p}{\mu_{i} \mu_{s} \mu_{V}}$ be the basic reproductive number of the hepatitis $C$ infection. Then

(1) if $R_{0} \leq 1, E_{0}=\left(\frac{\beta_{s}}{\mu_{s}}, 0,0,0\right)$ is the only equilibrium point of system (1) in $\Omega$ corresponding to a healthy individual, which is globally asymptotically stable.

(2) if $R_{0}>1$, there are two equilibrium points of system (1) in $\Omega, E_{0}$ which is unstable and the endemic equilibrium $E_{1}=\left(\frac{\beta_{s}}{k V^{*}+\mu_{s}}, \frac{\mu_{v} V^{*}}{p}\right.$, $\left.V^{*} \frac{\beta_{T} T_{\max } V^{*}}{\beta_{T} V^{*}+\mu_{T} T_{\max }}\right)$, which is locally asymptotically stable.

In the previous theorem, $V^{*}$ is the positive root of the equation

$$
A\left(V^{*}\right)^{2}+B V^{*}+C
$$

with

$$
\begin{aligned}
& A=k \beta_{T} \mu_{V}\left(\delta T_{\max }+\mu_{i}\right) \\
& B=-k \beta_{s} \beta_{T} p+\delta \beta_{T} \mu_{s} \mu_{v} T_{\max }+k \mu_{i} \mu_{v} \mu_{T} T_{\max }+\beta_{T} \mu_{i} \mu_{s} \mu_{v} \\
& C=\mu_{i} \mu_{s} \mu_{v} \mu_{T} T_{\max }-k \beta_{s} p \mu_{T} T_{\max }
\end{aligned}
$$

and such root exists when $R_{0}>1$. 


\section{Treatment}

One of the main advances in the treatment of hepatitis $\mathrm{C}$ is the combination of ribavirin with interferon- $\alpha$. The ribavirin is a drug that prevents the reproduction of hepatitis $\mathrm{C}$ virus, and simultaneously triggers an alert to the cells of the body's immune system to increase their defense mechanism against invading virus [9]. With the combination of pegylated interferon and ribavirin, the SVR rates (patients showing no traces of HCV after six months of treatment) can be achieved up (54-56)\% [3, 9].

Incorporating the combination therapy of pegylated interferon and ribavirin, system (1) becomes

$$
\begin{aligned}
& \dot{H}_{s}=\beta_{s}-k\left(1-n_{1}\right) H_{s} V-\mu_{s} H_{s}, \\
& \dot{H}_{i}=k\left(1-n_{1}\right) H_{s} V-\delta H_{i} T-\mu_{i} H_{i}, \\
& \dot{V}=p\left(1-\frac{n_{1}+n_{r}}{2}\right) H_{i}-\mu_{v} V, \\
& \dot{T}=\beta_{T} V\left(1-\frac{T}{T_{\max }}\right)-\mu_{T} T,
\end{aligned}
$$

where $n_{1}$ indicates the effectiveness (or efficacy) of interferon in blocking the replication of new infectious virions, while $n_{r}$ measures the efficacy of ribavirin to block the ability of the hepatitis $\mathrm{C}$ virus (HCV) to make more copies of itself. It should be mention that the therapy generally does not reach an efficiency $100 \%$, therefore we assume $0<n_{1}<1$. The term $\left(\frac{n_{1}+n_{r}}{2}\right)$ represents the effectiveness of the combined effect of interferon and ribavirin. From now on, we assume that $0<n_{r}<1$ and so $0<\frac{n_{1}+n_{r}}{2}<1$ 


\section{Optimal Control Model with Immune System}

The combination therapy of interferon and ribavirin is expensive and has side effects such as hemolytic anaemia, somnolence, stomach disturbances, shortness of breath, among others (http://www.medicine.net.com). There is a necessity to find more effective drugs with less secondary effect. In this section, we present an alternative for better treatment dosage regimen using control theory.

\subsection{Control problem statement}

To achieve optimal treatment according to the theory we define the cost functional

$$
J\left(n_{1}, n_{r}\right)=\int_{0}^{t_{f}}\left[\frac{1}{2} C_{1} V^{2}+\left(\frac{1}{2} C_{2} n_{1}^{2}+\frac{1}{2} C_{3} n_{r}^{2}\right)\right] d t, \quad C_{i}>0, \quad i=1, \ldots, 3 .
$$

The first term represents the main biological target, i.e., reducing the viral load, while the other two terms consist of the cost of treatment. The constant $C_{1}$ represents the cost associated with viral load reduction, while $C_{2}$ and $C_{3}$ are costs related to the drugs side effects. Drugs administration in general produces highly toxicity to the human body, and for this reason we adopt a second-degree control instead of a linear one.

The objective is to find an optimal pair $\left(n_{1}^{*}, n_{r}^{*}\right)$ such that

$$
J\left(n_{1}^{*}, n_{r}^{*}\right)=\min \left\{J\left(n_{1}, n_{r}\right):\left(n_{1}, n_{r}\right) \in \mathfrak{N}\right\},
$$

where

$$
\mathfrak{N}=\left\{\left(n_{1}, n_{r}\right) / 0 \leq n_{1}, n_{r} \leq 1: n_{1}, n_{r} \text { measurable functions }\right\}
$$

is the set of controls. 
The Lagrangian asociate to the problem control is given by

$\mathcal{L}\left(H_{s}(t), H_{i}(t), V(t), T(t), u_{1}(t), u_{r}(t), t\right)=\left[\frac{1}{2} C_{1} V^{2}+\left(\frac{1}{2} C_{2} n_{1}^{2}+\frac{1}{2} C_{3} n_{r}^{2}\right)\right]$.

Thus, solving the problem of control consists to minimize

$$
J[u]
$$

subject to

$$
\dot{x}(t)=\left(\dot{H}_{s}(t), \dot{H}_{i}(t), \dot{V}(t), T(t)\right),
$$

with the boundary conditions

$$
x_{0}=x(0)=\left(H_{s}^{0}, H_{i}^{0}, V^{0}, T^{0}\right), x(T), u=\left(n_{1}, n_{r}\right) \in \mathfrak{N} .
$$

A pair $\left(x_{0},\left(n_{1}, n_{r}\right)\right)$ is admissible, if it solves the control problem. The set of admissible pairs is called admissible set and is denoted by $\mathcal{F}$.

\subsection{Existence of optimal control}

Theorem 3. Consider the posed control problem given above, with $\mathcal{L}$ continuous, then there exists $u^{*}=\left(n_{1}^{*}, n_{r}^{*}\right) \in \mathfrak{N}$ such that

$$
J\left[n_{1}^{*}, n_{r}^{*},\right]=\min \left\{J\left[n_{1}, n_{r}\right]: u=\left(n_{1}, n_{r}\right) \in \mathfrak{N}\right\} .
$$

Proof. To prove that there is an optimal control, we use a result of Fleming and Rishel ([10], Theorem 4.1 and its corollary, p. 68-69). Specifically, we have to prove the following:

(a) The set of admissible pairs $\mathcal{F}$ is not empty.

(b) The control set $\mathfrak{N}$ is convex and closed.

(c) The vector field $f$ of system (3) can be written as $f(t, x, u)=\alpha(t, x)$ $+\beta(t, x) u$.

(d) The Lagrangian $\mathcal{L}$ is convex in $\mathfrak{N}$. 
(e) There are constant $c_{1}, c_{2}>0$ and $\beta>1$ such that

$$
(t, x, u) \geq c_{1}|u|^{\beta}-c_{2} .
$$

It is clear that the solutions of system (3) are bounded, then according to a result of Lukes ([11], Theorem 9.2.1) there exists an admissible pair $\left(x_{0}, u\right) \in \mathcal{F}$, therefore $\mathcal{F} \neq \phi$ and condition (a) holds.

By definition $\mathfrak{N}$ is convex and bounded, thus condition (b) is fulfilled and the condition (d) is satisfied since the Lagrangian $\mathcal{L}$ is a quadratic function of $u$.

To prove (c), we notice that system (3) can be written as

$$
\left(\begin{array}{c}
\dot{H}_{s} \\
\dot{H}_{i} \\
\dot{V} \\
\dot{T}
\end{array}\right)=\left(\begin{array}{c}
\beta_{s}-k H_{s} V-\mu_{s} H_{s} \\
k H_{s} V-\delta H_{i} T-\mu_{i} H_{i} \\
p H_{i}-\mu_{v} V \\
\beta_{T}\left(1-\frac{T}{T_{\max }}\right) V-\mu_{T} T
\end{array}\right)+\left(\begin{array}{cc}
k H_{s} V & 0 \\
-k H_{s} V & 0 \\
-\frac{p H_{i}}{2} & -\frac{p H_{i}}{2} \\
0 & 0
\end{array}\right)\left(\begin{array}{l}
n_{1} \\
n_{r}
\end{array}\right) .
$$

Only remains to prove (e). For this, we notice that $0<n_{1}, n_{r}<1$ and $c_{1}, c_{2}>0$, then

$$
c_{1} n_{1}^{2} \leq c_{1} n_{1} \leq c_{1}
$$

and

$$
c_{2} n_{r}^{2} \leq c_{2} n_{r} \leq c_{2}
$$

Consequently,

$$
c_{1} n_{1}^{2}+c_{2} n_{r}^{2}-\left(c_{1}+c_{2}\right) \leq 0
$$

If we define $d_{1}=\min \left\{c_{1}, c_{2}\right\}$ and $d_{2}=c_{1}+c_{2}$, then

$$
d_{1}\left(n_{1}^{2}+n_{r}^{2}\right)-d_{2} \leq 0
$$


Thus

$\mathcal{L}\left(H_{s}(t), H_{i}(t), V(t), T(t), u(t), t\right)=\frac{1}{2}\left[C_{1} V^{2}(t)+C_{2} n_{1}^{2}(t)+c_{3} n_{r}^{2}\right] \geq 0 \geq d_{1}|u|^{2}-d_{2}$, and condition (e) holds with $c_{1}=d_{1}, c_{2}=d_{2}$, and $\beta=2$.

Therefore, by the results proved above, there exists $\left(x_{0}, u^{*}(t)\right)$ which minimizes $\mathcal{J}\left(x_{0}, u\right)$ in $\mathcal{F}$.

\subsection{Characterization of optimal control}

We use the maximum principle of Pontryagin $[16,10]$, to derive the necessary conditions to control problem.

Theorem 4. Given an optimal pair $\left(n_{1}^{*}, n_{r}^{*}\right)$, and solutions $\left(H_{s}, H_{i}, T, V\right)$ of system (3), there are continuous functions $\lambda_{i}(t), i=1, \ldots, 4$ satisfying

$$
\begin{aligned}
& \dot{\lambda}_{1}=k\left(1-n_{1}\right) V\left(\lambda_{1}-\lambda_{2}\right)-\mu_{s} \lambda_{1}, \\
& \dot{\lambda}_{2}=\left(\delta T+\mu_{i}\right) \lambda_{2}-p\left(1-\frac{n_{1}+n_{r}}{2}\right) \lambda_{3}, \\
& \dot{\lambda}_{3}=-C_{1} V+k\left(1-n_{1}\right) H_{s}\left(\lambda_{1}-\lambda_{2}\right)+\mu_{v} \lambda_{3}-\beta_{T}\left(1-\frac{T}{T_{\max }}\right) \lambda_{4}, \\
& \dot{\lambda}_{4}=\delta H_{i} \lambda_{2}+\mu_{T} \lambda_{4}+\frac{\beta_{T} V}{T_{\max }} .
\end{aligned}
$$

Additionally, $u^{*}=\left(n_{1}^{*}, n_{r}^{*}\right)$ is characterized by

$$
n_{1}^{*}=\min \left\{\max \left\{0,-\frac{1}{C_{2}}\left[k H_{s} V\left(\lambda_{1}-\lambda_{2}\right)+\frac{1}{2} p H_{i} \lambda_{3}\right]\right\}, 1\right\}
$$

and

$$
n_{r}^{*}=\min \left\{\max \left\{0, \frac{p H_{i} \lambda_{3}}{2 C_{3}}\right\}, 1\right\} .
$$


Proof. The Hamiltonian associated with the control problem is

$$
\begin{aligned}
H\left(H_{s}, H_{i},\right. & \left.V, T, \lambda_{1}, \lambda_{2}, \lambda_{3}, \lambda_{4}, n_{1}, n_{r}, t\right) \\
= & {\left[\frac{1}{2} C_{1} V^{2}+\left(\frac{1}{2} C_{2} n_{1}^{2}+\frac{1}{2} C_{3} n_{r}^{2}\right)\right] } \\
& +\lambda_{1}\left[\beta_{s}-k\left(1-n_{1}\right) H_{s} V-\mu_{s} H_{s}\right] \\
& +\lambda_{2}\left[k\left(1-n_{1}\right) H_{s} V-\delta H_{i}-T \mu_{i} H_{i}\right] \\
& +\lambda_{3}\left[p\left(1-\frac{n_{1}+n_{r}}{2}\right) H_{i}-\mu_{v} V\right] \\
& +\lambda_{4}\left[\beta_{T} V\left(1-\frac{T}{T_{\max }}\right)-\mu_{T} T\right] \\
& +v_{1} n_{1}-v_{2}\left(1-n_{1}\right)+w_{1} n_{r}-w_{2}\left(1-n_{r}\right),
\end{aligned}
$$

where $v_{1}(t), v_{2}(t), w_{1}(t), w_{2}(t) \geq 0$, are penalty multipliers that guarantee that $n_{1}, n_{r} \in[0,1]$ and satisfy

$$
v_{1} n_{1}^{*}=0, \quad v_{2}\left(1-n_{1}^{*}\right)=0, \quad w_{1} n_{r}^{*}=0, \quad w_{2}\left(1-n_{r}^{*}\right)=0 .
$$

By Pontryagin theorem, $\lambda_{i}$ satisfy

$$
\begin{aligned}
& \dot{\lambda}_{1}=-\frac{\partial H}{\partial H_{s}}=k\left(1-n_{1}\right) V\left(\lambda_{1}-\lambda_{2}\right)+\mu_{s} \lambda_{1}, \\
& \dot{\lambda}_{2}=-\frac{\partial H}{\partial H_{i}}=\left(\delta T+\mu_{i}\right) \lambda_{2}-p\left(1-\frac{n_{1}+n_{r}}{2}\right) \lambda_{3}, \\
& \dot{\lambda}_{3}=-\frac{\partial H}{\partial V}=-C_{1} V+k\left(1-n_{1}\right) H_{s}\left(\lambda_{1}-\lambda_{2}\right)+\mu_{v} \lambda_{3}-\beta_{T}\left(1-\frac{T}{T_{\max }}\right) \lambda_{4}, \\
& \dot{\lambda}_{4}=-\frac{\partial H}{\partial T}=\delta H_{i} \lambda_{2}+\mu_{T} \lambda_{4}+\frac{\beta_{T} V}{T_{\max }}
\end{aligned}
$$

with transversality conditions $\lambda_{i}(T)=0, i=1, \ldots, 4$. 
To characterize $n_{1}^{*}$, we use the optimality condition

$$
\frac{\partial H\left(n_{1}^{*}\right)}{\partial n_{1}}=0
$$

Notice that

$$
\frac{\partial H\left(n_{1}^{*}\right)}{\partial n_{1}}=C_{2} n_{1}^{*}+k H_{s} V \lambda_{1}-k H_{s} V \lambda_{2}-\frac{1}{2} p H_{i} \lambda_{3}+v_{1}+v_{2}
$$

then

$$
n_{1}^{*}=-\frac{1}{C_{2}}\left[k H_{s} V\left(\lambda_{1}-\lambda_{2}\right)+\frac{1}{2} p H_{i} \lambda_{3}+\left(v_{1}+v_{2}\right)\right] .
$$

Similarly the optimality condition

$$
\frac{\partial H\left(n_{r}^{*}\right)}{\partial n_{r}}=0
$$

gives

$$
n_{r}^{*}=\frac{p H_{i} \lambda_{3}-\left(w_{1}+w_{2}\right)}{2 C_{3}} .
$$

To obtain an explicit expression for the optimal controls $n_{1}^{*}$ and $n_{r}^{*}$, we proceed as follows:

For $n_{1}^{*}$, we consider the following cases:

- If $0<n_{1}^{*}(t)<1$, then from (7), $v_{1}(t)=0=v_{2}(t)$, and this implies that

$$
n_{1}^{*}(t)=-\frac{1}{C_{2}}\left[k H_{s} V\left(\lambda_{1}-\lambda_{2}\right)+\frac{1}{2} p H_{i} \lambda_{3}\right]
$$

- If $n_{1}^{*}=0$, then from (7), $v_{2}=0, v_{1} \geq 0$ and

$$
0=n_{1}^{*}(t)=-\frac{1}{C_{2}}\left[k H_{s} V\left(\lambda_{1}-\lambda_{2}\right)+\frac{1}{2} p H_{i} \lambda_{3}+v_{1}\right] .
$$


Since $v_{1}(t) \geq 0$,

$$
\frac{1}{C_{2}}\left[k H_{s} V\left(\lambda_{1}-\lambda_{2}\right)+\frac{1}{2} p H_{i} \lambda_{3}\right] \leq 0 .
$$

- If $n_{1}^{*}(t)=1$, from (7) we get $v_{1}(t)=0, v_{2} \geq 0$, and in this case

$$
1=n_{1}^{*}(t)=-\frac{1}{C_{2}}\left[k H_{s} V\left(\lambda_{1}-\lambda_{2}\right)+\frac{1}{2} p H_{i} \lambda_{3}+v_{2}\right]
$$

implying

$$
1=-\frac{1}{C_{2}}\left[k H_{s} V\left(\lambda_{1}-\lambda_{2}\right)+\frac{1}{2} p H_{i} \lambda_{3}\right]-\frac{v_{2}}{C_{2}}
$$

Since $v_{2} \geq 0$, we conclude that

$$
-\frac{1}{C_{2}}\left[k H_{s} V\left(\lambda_{1}-\lambda_{2}\right)+\frac{1}{2} p H_{i} \lambda_{3}\right] \geq 1
$$

Joining the three previous cases, $n_{1}^{*}$ is completely characterized and

$$
n_{1}^{*}(t)=\min \left\{\max \left\{0,-\frac{1}{C_{2}}\left[k H_{s} V\left(\lambda_{1}-\lambda_{2}\right)+\frac{1}{2} p H_{i} \lambda_{3}\right]\right\}, 1\right\} .
$$

Proceeding similarly for $n_{r}^{*}$, we obtain

$$
n_{r}^{*}=\min \left\{\max \left\{0, \frac{p H_{i} \lambda_{3}}{2 C_{3}}\right\}, 1\right\} .
$$

To prove the uniqueness of the optimal control, we will use the fact that the solutions of the system (3) and the adjoint equations are bounded.

Theorem 5. For $t_{f}$ small enough, the bounded optimality system solutions are unique.

See Appendix A for the proof. 


\section{Numerical Results}

In this section, we present numerical simulations of system (3) with no treatment, fixed dose treatment, and optimal treatment. To find the optimal solutions, we estimate the optimal controls $n_{1}^{*}$ and $n_{r}^{*}$ with the method of forward-back sweep Runge Kutta of fourth order programmed in MATLAB. The parameters used in the simulations are given in Table 1.

Table 1. Demographic and epidemiological parameters. The values are taken from ([1], [15]). For these parameters we have $R_{0}=2.4$

\begin{tabular}{|c|c|c|c|}
\hline Parameter & Value & Parameter & Value \\
\hline$\beta_{s}$ & 100 & $p$ & 200 \\
$k$ & 0.00006 & $\mu_{s}$ & 0.02 \\
$\mu_{i}$ & 5 & $\mu_{v}$ & 5 \\
$\delta$ & 0.00001 & $\beta_{T}$ & 0.0003 \\
$\mu_{T}$ & 0.02 & $T_{\max }$ & 1200 \\
\hline
\end{tabular}




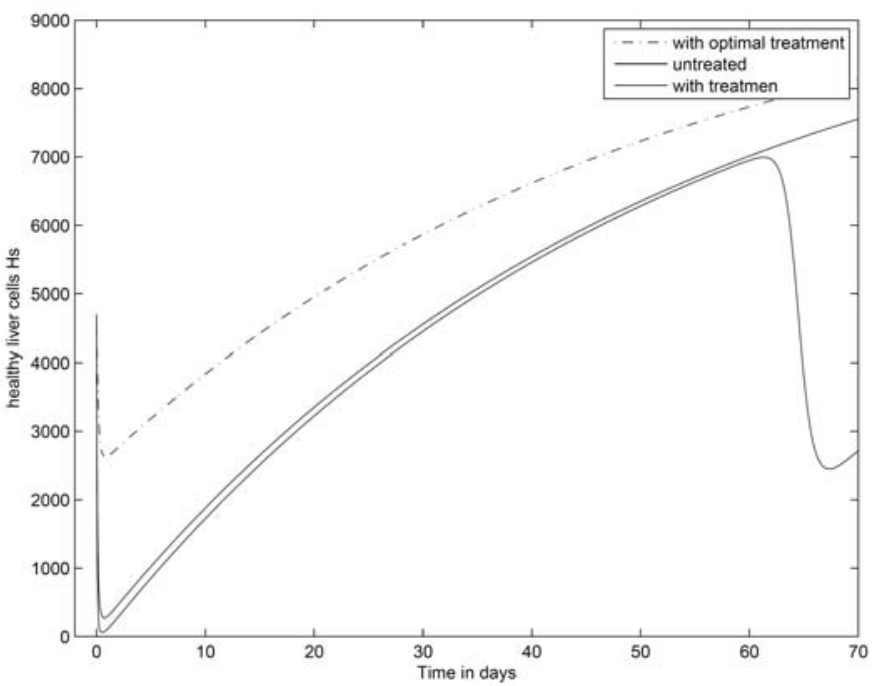

(A) Healthy liver cells

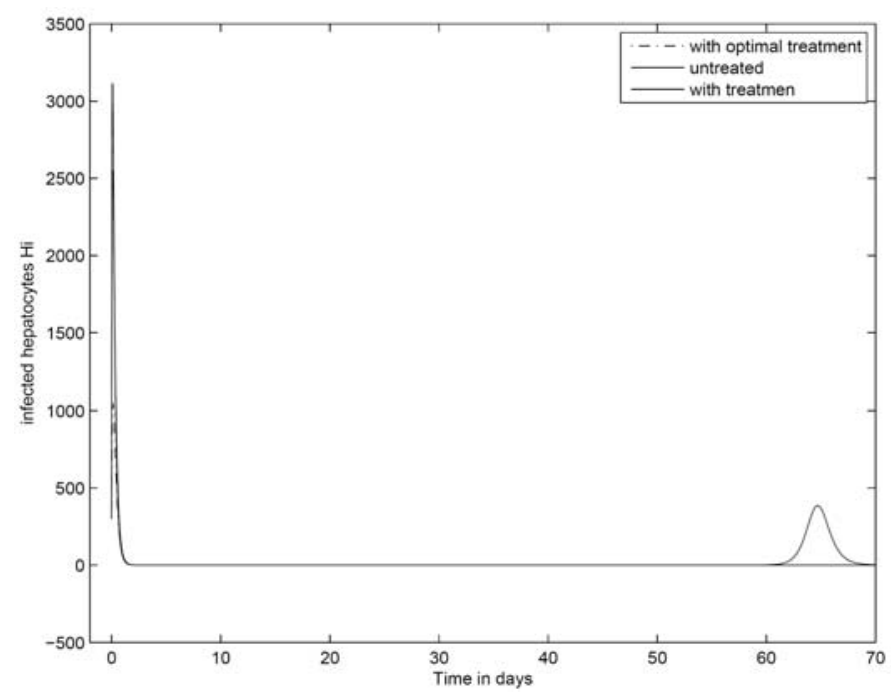

(B) Infected hepatocytes

Figure 1. Comparison of the dynamics of healthy and infected hepatocytes with no treatment, treatment, and optimal treatment. With $R_{0}=2.4, C_{1}=0.1, C_{2}=0.001, C_{3}=0.001$, efficacy of interferon $n_{1}=0.4$ and ribavirin $n_{r}=0.3$. 


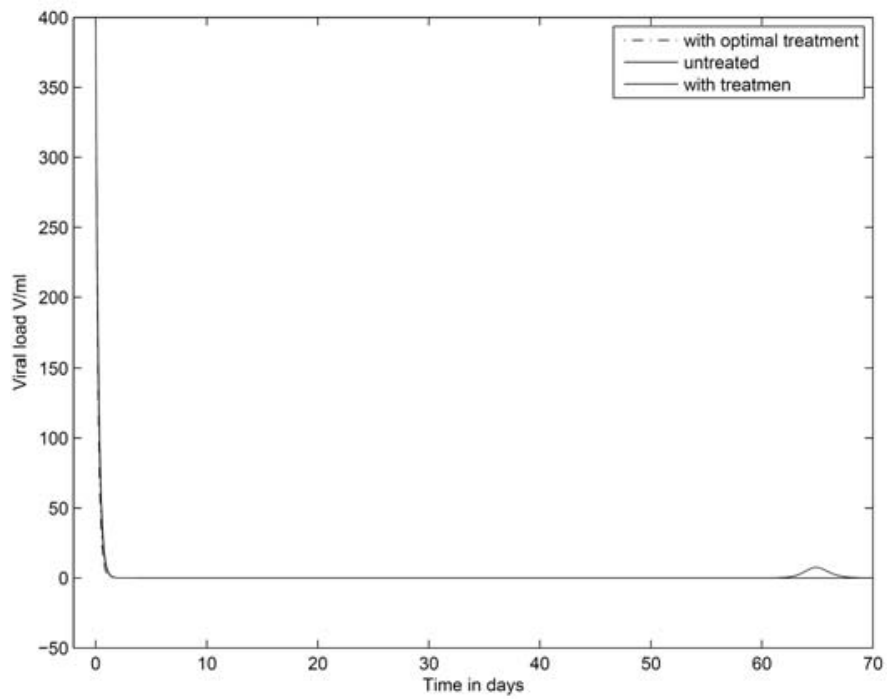

(A) Viral load

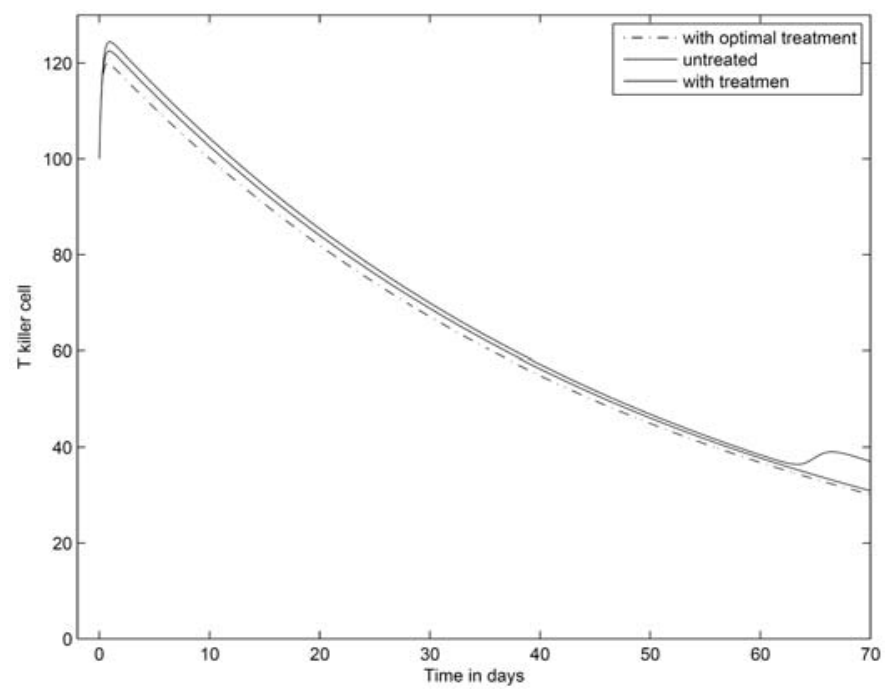

(B) $T$ killer cell

Figure 2. Dynamics of viral load and $T$ killer cell with no treatment, treatment, and optimal treatment. With $R_{0}=2.4, C_{1}=0.1, C_{2}=0.001$, $C_{3}=0.001$, efficacy of interferon $n_{1}=0.4$ and ribavirin $n_{r}=0.3$. 


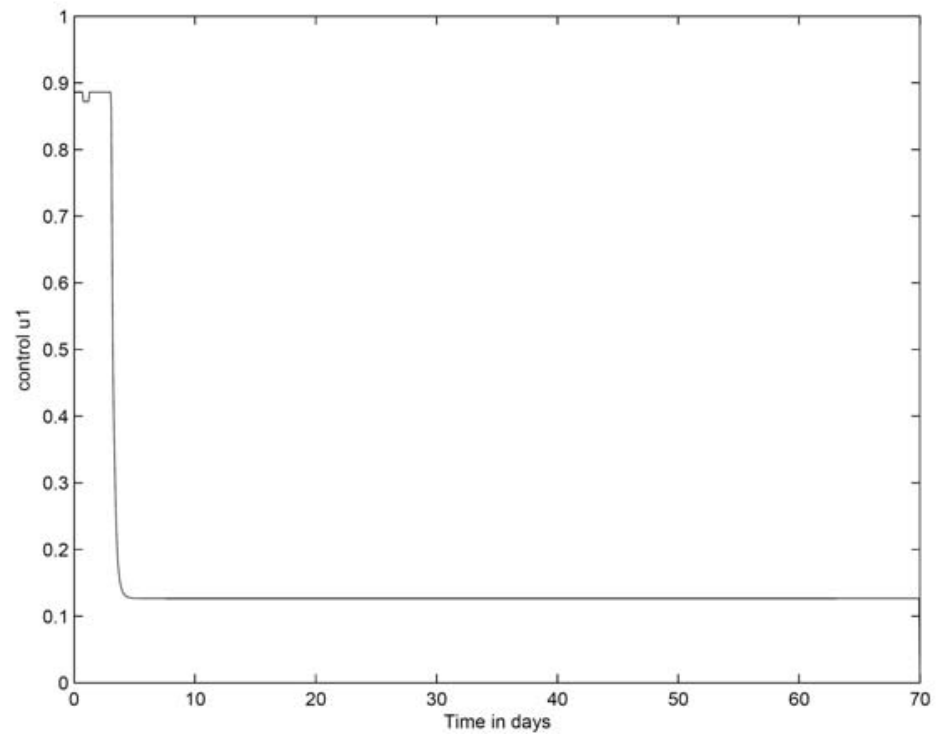

(A) Optimal control $n_{1}$

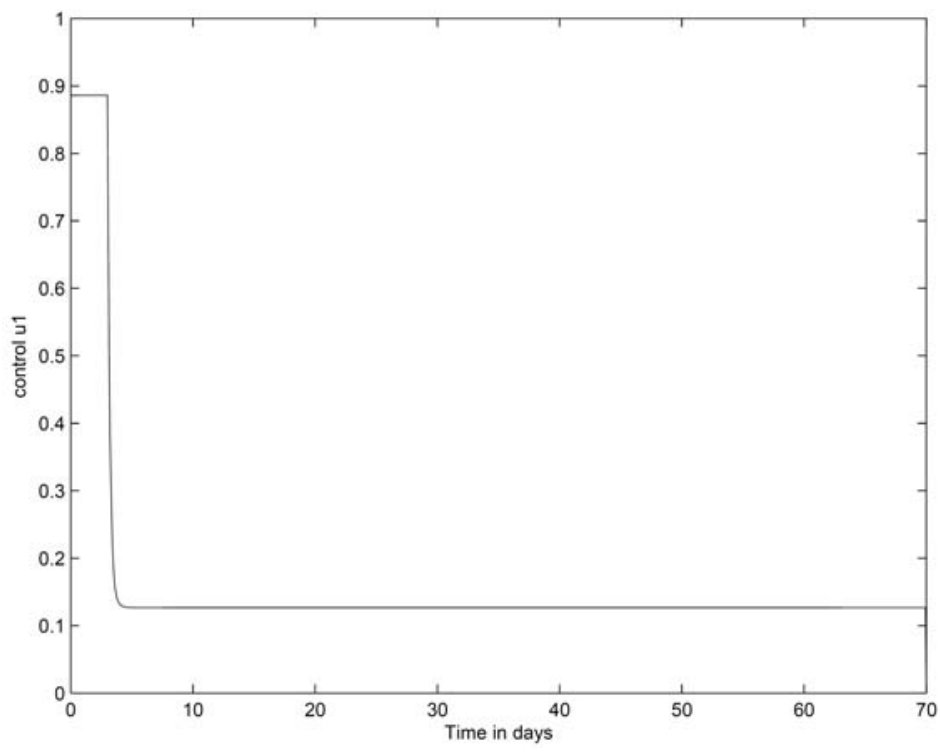

(B) Optimal control $n_{r}$

Figure 3. Comparison of the dynamic of optimal controls $n_{1}$ and $n_{r}$. 
Figures 1 and 2 show the temporal course of the dynamical variables $H_{s}, H_{i}, V$, and $T$. We notice that with optimal treatment the number of healthy hepatocites recover faster than with the usual treatment. Also, without treatment healthy hepatocytes oscillate to an endemic state less than $\frac{\beta_{s}}{\mu_{s}}$ due to the fact that $R_{0}>1$. As $R_{0}$ increases the differences among the three scenarios are more pronounced. This shows that the response of the patient to treatment with optimal therapy combined ribavirin and interferon is more successful than the therapy in which drug dose is fixed all the time of treatment.

In Figures 1(B) and 2(A), we observe that the temporal course of the infected hepatocites and viral load is similar, in both cases there is a fast decrease when treatment is applied, and an oscillatory behaviour approaching the endemic state when therapy is not applied. However, the temporal dynamics of the optimal controls $n_{1}^{*}, n_{r}^{*}$ illustrated in Figure 3 show that a high efficacy (and therefore more drug doses) is needed only during the first five days of treatment, but afterwards dosis should be decreased. This coincides with the results given in $([4,15])$, which suggest that high doses should be administrated until the patient achieves a notorious progress, after the doses should be reduced in order to avoid excessive economical costs and secondary effects.

In Figure 2(B), we notice that virus-specific $T$ cells first increase to a maximum due to the presence of $\mathrm{HCV}$, since $T$ cells start to eliminate infected macrophagues, consequently the viral load is reduced and they start to decline. With no treatment, we observe oscillations of $T$ cells following the dynamics of virus and infected macrophagues. With optimal treatment, the decline of $T$ cells is steeper in comparison with the decline under monotherapy. This behaviour suggest that symptoms associated with the immune system action can be reduced with optimal treatment. 
Our model does not show a biphasic or triphasic decline during the period of high dosage administration, on the contrary, the decline of viral load is very pronounced all the time. This behaviour probably is due to the joint action of the immune system and medication. Thus, patients with a strong immune system are more likely to reduce viral load faster during the treatment.

Several simulations were made for small values of $C_{2}$ and $C_{3}$, and we note that in the case of drugs with fewer side effects the period of time in which the drug doses is high. This is, they remain high during the first five days, and then they fall to a levels very close to zero. This is consistent with the fact that high doses are required until the viral load begins to decrease to virtually undetectable levels.

\section{Conclusion}

In this work, we presented a model for the dynamics of the hepatitis $\mathrm{C}$ virus taking into account healthy and infected hepatocytes, virus load, and $T$ cells. We incorporated treatment with interferon and ribavirin, and we use the deterministic control theory to find an optimal treatment strategy. For this problem, we assume that drugs efficacy is proportional to drugs dosage.

We proved the existence and uniqueness of the optimal control which gives consistency to our results. The optimal treatment caused viral load to go down at the same level that therapy with fixed dose, but with the difference that the optimal therapy also diminish the side effects caused by the treatment.

\section{Acknowledgement}

L. Esteva acknowledges support from project IN112713, PAPIITUNAM. A. Peregrino and G. Blé acknowledge support from project CB2012-181247. A. Peregrino acknowledges support from project FORDECyT-265667. 


\section{References}

[1] R. Avendaño, L. Esteva, J. A. Flores, J. L. Fuentes Allen, J. Gómez and Je. LópezEstrada, A mathematical model for the dynamics of hepatitis C, Journal of Theoretical Medicine 4(2) (2002), 109-118.

[2] S. Banerjee, R. Keval and S. Gakkhar, Modeling the dynamics of hepatitis C virus with combined antiviral drug therapy: Interferon and Ribavirin, Mathematical Biosciences 245(2) (2013) 235-248.

DOI: https://doi.org/10.1016/j.mbs.2013.07.005

[3] S. Crotty, C. E. Cameron and R. Andino, RNA virus error catastrophe: Direct molecular test by using ribavirin, Proc. Natl. Acad. Sci. USA 98(12) (2001), 6895-6900.

DOI: https://doi.org/10.1073/pnas.111085598

[4] S. P. Chakrabarty, Optimal efficacy of ribavirin in the treatment of hepatitis C, Optimal Control Applications and Methods 30(6) (2009), 594-600.

DOI: https://doi.org/10.1002/oca.894

[5] S. P. Chakrabarty and H. R. Joshi, Optimally controlled treatment strategy using interferon and ribavirin for hepatitis C, Journal of Biological Systems 17(1) (2009) 97-110.

DOI: https://doi.org/10.1142/S0218339009002727

[6] N. M. Dixit, J. E. Layden-Almer, T. J. Layden and A. S. Perelson, Modelling how ribavirin improves interferon response rates in hepatitis $\mathrm{C}$ virus infection, Nature 432 (2004), 922-924.

DOI: https://doi.org/10.1038/nature03153

[7] H. Dahari, A. Lo, R. M. Ribeiro and A. S. Perelson, Modelling hepatitis C virus dynamics: Liver regeneration and critical drug efficacy, Journal of Theoretical Biology 247(2) (2007), 371-381.

DOI: https://doi.org/10.1016/j.jtbi.2007.03.006

[8] H. Dahari, R. M. Ribeiro and A. S. Perelson, Triphasic decline of hepatitis C virus RNA during antiviral therapy, Hepatology 46(1) (2007), 16-21.

DOI: https://doi.org/10.1002/hep.21657

[9] J. Emilio, Hepatitis C Virus Disease: Immunobiology and Clinical Applications, Springer, 2008.

[10] W. Fleming and R. Rishel, Deterministic and Stochastic Optimal Controls, SpringerVerlag, 1975.

[11] L. D. Lukes, Differential Equations: Classical to Controlled, Vol. 162 of Mathematics in Science and Engineering, Academic Press, New York, 1982.

[12] S. Lenhart and J. T. Workman, Optimal Control Applied to Biological Models, Mathematical and Computational Biology Series, Chapman and Hall/CRC, 2007. 
[13] N. K. Martin, A. B. Pitcher, P. Vickerman, A. Vassal and M. Hickman, Optimal control of hepatitis $\mathrm{C}$ antiviral treatment programme delivery for prevention amongst a population of injecting drug users, PLoS ONE 6(8) (2011), e22309.

DOI: https://doi.org/10.1371/journal.pone.0022309

[14] J. M. Ntaganda, M. S. D. Haggar and B. Mampassi, Fuzzy logic strategy for solving an optimal control problem of therapeutic hepatitis C virus dynamics, Open Journal of Applied Sciences 5(9) (2015), 527-541.

DOI: https://doi.org/10.4236/ojapps.2015.59051

[15] A. U. Neumann, N. P Lam, H. Dahari, D. R. Gretch, T. E. Wiley, T. J. Layden and A. S. Perelson, Hepatitis $\mathrm{C}$ viral dynamics in vivo and the antiviral efficacy of interferon-alpha therapy, Science 282(5386) (1998), 103-107.

DOI: http://dx.doi.org/10.1126/science.282.5386.103

[16] L. S. Pontryagin, V. G. Boltyanskii, R. V. Gamkrelidze and E. F. Mishchenko, The mathematical theory of optimal process, Translated from de Russian by K. N. Trirogoff; Edited by L. W. Neustadt, Interscience Publisher, John Wiley and Sons, Inc., New York, London, 1962.

[17] H. R. Roson, Clinical practice. Cronic hepatitis C infection, The New England Journal of Medicine 364(25) (2011), 2429-2438.

DOI: http://dx.doi.org/10.1056/NEJMcp1006613

[18] World Health Organization, Hepatitis C-global prevalence (update), World Health Org. Weekly Epidemiol. Rec. 75 (2000), 18-19.

[19] S. Zeuzem and E. Hermann, Dynamics of hepatitis C virus infection, Annals of Hepatology 1(2) (2002), 56-63. 


\section{Appendix A: Uniqueness of Optimal Control}

Proof of Theorem 5. Let $\left(H_{s}, H_{i}, V, T, \lambda_{1}, \lambda_{2}, \lambda_{3}, \lambda_{4}\right)$ and $\left(\bar{H}_{s}, \bar{H}_{i}, \bar{V}, \bar{T}, \bar{\lambda}_{1}, \bar{\lambda}_{2}, \bar{\lambda}_{3}, \bar{\lambda}_{4}\right)$ two different optimality system solutions.

We assume that there exist $\alpha>0$ and functions $r_{i}(t), g_{i}(t), r, j=1$, $\ldots, 4$, such that

$$
\begin{aligned}
& H_{s}(t)=e^{\alpha t} q_{1}(t), \quad H_{i}(t)=e^{\alpha t} q_{2}(t), \quad V(t)=e^{\alpha t} q_{3}(t), \quad T(t)=e^{\alpha t} q_{4}(t), \\
& \lambda_{1}(t)=e^{-\alpha t} r_{1}(t), \quad \lambda_{2}(t)=e^{-\alpha t} r_{2}(t), \quad \lambda_{3}(t)=e^{-\alpha t} r_{3}(t), \quad \lambda_{4}(t)=e^{-\alpha t} r_{4}(t),
\end{aligned}
$$

and

$$
\begin{aligned}
& \bar{H}_{s}(t)=e^{\alpha t} \bar{q}_{1}(t), \quad \bar{H}_{i}(t)=e^{\alpha t} \bar{q}_{2}(t), \quad \bar{V}(t)=e^{\alpha t} \bar{q}_{3}(t), \quad \bar{T}(t)=e^{\alpha t} \bar{q}_{4}(t), \\
& \bar{\lambda}_{1}(t)=e^{-\alpha t} \bar{r}_{1}(t), \quad \bar{\lambda}_{2}(t)=e^{-\alpha t} \bar{r}_{2}(t), \quad \bar{\lambda}_{3}(t)=e^{-\alpha t} \bar{r}_{3}(t), \quad \bar{\lambda}_{4}(t)=e^{-\alpha t} \bar{r}_{4}(t),
\end{aligned}
$$

for $t$ sufficiently small. Additionally, let

$$
\begin{gathered}
n_{1}^{*}=\min \left\{\max \left\{0,-\frac{1}{C_{2}}\left[k H_{s} V \lambda_{1}-k H_{s} V \lambda_{2}+\frac{1}{2} p H_{i} \lambda_{3}\right]\right\}, 1\right\}, \\
\bar{n}_{1}^{*}=\min \left\{\max \left\{0,-\frac{1}{C_{2}}\left[k \bar{H}_{s} \bar{V} \bar{\lambda}_{1}-k \bar{H}_{s} \bar{V} \bar{\lambda}_{2}+\frac{1}{2} p \bar{H}_{i} \bar{\lambda}_{3}\right]\right\}, 1\right\},
\end{gathered}
$$

and

$$
\begin{aligned}
& n_{r}^{*}=\min \left\{\max \left\{0, \frac{p H_{i}}{2 C_{3}}\right\}, 1\right\}, \\
& \bar{n}_{r}^{*}=\min \left\{\max \left\{0, \frac{p \bar{H}_{i}}{2 C_{3}}\right\}, 1\right\},
\end{aligned}
$$

the controls associated with the above optimality systems given by (9) and (11), respectively. 
Substituting the solutions given by (14) and (15) in the optimality system composed by the state and adjoin equations and the controls, we get

$$
\begin{aligned}
& \dot{q}_{1}+\alpha q_{1}=e^{-\alpha t} \beta_{s}-k\left(1-n_{1}^{*}\right) e^{\alpha t} q_{1} q_{3}-\mu_{s} q_{1}, \\
& \dot{q}_{2}+\alpha q_{2}=k\left(1-n_{1}^{*}\right) e^{\alpha t} q_{1} q_{3}-\delta e^{\alpha t} q_{2} q_{4}-\mu_{i} q_{2}, \\
& \dot{q}_{3}+\alpha q_{3}=p\left(1-\frac{n_{1}^{*}+n_{r}^{*}}{2}\right) q_{2}-\mu_{v} q_{4}, \\
& \dot{q}_{4}+\alpha q_{4}=\beta_{T}\left(1-\frac{e^{\alpha t} q_{4}}{T_{\max }}\right) q_{3}-\mu_{T} q_{4}, \\
& \dot{r}_{1}-\alpha r_{1}=k\left(1-n_{1}^{*}\right) e^{\alpha t} q_{3} r_{1}-k\left(1-n_{1}^{*}\right) e^{\alpha t} q_{3} r_{2}+\mu_{s} r_{1}, \\
& \dot{r}_{2}-\alpha r_{2}=\delta e^{\alpha t} q_{4} r_{2}-\mu_{i} r_{2}-p\left(1-\frac{n_{1}^{*}+n_{r}^{*}}{2}\right) r_{3}, \\
& \dot{r}_{3}-\alpha r_{3}=-C_{1} e^{2 \alpha t} q_{3}+k\left(1-n_{1}^{*}\right) e^{\alpha t}\left(q_{1} r_{1}-q_{1} r_{2}\right)+\mu_{v} r_{3}-\beta_{T}\left(1-\frac{e^{\alpha t} q_{4}}{T_{\max }}\right) r_{4}, \\
& \dot{r}_{4}-\alpha r_{4}=\delta e^{\alpha t} q_{2} r_{2}+\mu_{T} r_{4}+\frac{\beta_{T}}{T_{\max }} e^{\alpha t} q_{3} r_{4},
\end{aligned}
$$

and

$$
\begin{aligned}
& \dot{\bar{q}}_{1}+\alpha \bar{q}_{1}=e^{-\alpha t} \beta_{s}-k\left(1-n_{1}^{*}\right) e^{\alpha t} \bar{q}_{1} \bar{q}_{3}-\mu_{s} \bar{q}_{1}, \\
& \dot{\bar{q}}_{2}+\alpha \bar{q}_{2}=k\left(1-n_{1}^{*}\right) e^{\alpha t} \bar{q}_{1} \bar{q}_{3}-\delta e^{\alpha t} \bar{q}_{2} \bar{q}_{4}-\mu_{i} \bar{q}_{2}, \\
& \dot{\bar{q}}_{3}+\alpha \bar{q}_{3}=p\left(1-\frac{n_{1}^{*}+n_{r}^{*}}{2}\right) \bar{q}_{2}-\mu_{v} \bar{q}_{4}, \\
& \dot{\bar{q}}_{4}+\alpha \bar{q}_{4}=\beta_{T}\left(1-\frac{e^{\alpha t} \bar{q}_{4}}{T_{\max }}\right) \bar{q}_{3}-\mu_{T} \bar{q}_{4}, \\
& \dot{\bar{r}}_{1}-\alpha \bar{r}_{1}=k\left(1-n_{1}^{*}\right) e^{\alpha t} \bar{q}_{3} \bar{r}_{1}-k\left(1-n_{1}^{*}\right) e^{\alpha t} \bar{q}_{3} \bar{r}_{2}+\mu_{s} \bar{r}_{1},
\end{aligned}
$$




$$
\begin{aligned}
& \dot{\bar{r}}_{2}-\alpha \bar{r}_{2}=\delta e^{\alpha t} \bar{q}_{4} \bar{r}_{2}-\mu_{i} \bar{r}_{2}-p\left(1-\frac{n_{1}^{*}+n_{r}^{*}}{2}\right) \bar{r}_{3} \\
& \dot{\bar{r}}_{3}-\alpha \bar{r}_{3}=-C_{1} e^{2 \alpha t} \bar{q}_{3}+k\left(1-n_{1}^{*}\right) e^{\alpha t}\left(\bar{q}_{1} \bar{r}_{1}-\bar{q}_{1} \bar{r}_{2}\right)+\mu_{v} \bar{r}_{3}-\beta_{T}\left(1-\frac{e^{\alpha t} \bar{q}_{4}}{T_{\max }}\right) \bar{r}_{4} \\
& \dot{\bar{r}}_{4}-\alpha \bar{r}_{4}=\delta e^{\alpha t} \bar{q}_{2} \bar{r}_{2}+\mu_{T} \bar{r}_{4}+\frac{\beta_{T}}{T_{\max }} e^{\alpha t} \bar{q}_{3} \bar{r}_{4} .
\end{aligned}
$$

Additionally by (12) and (13), we have

$$
\left(n_{1}^{*}-\bar{n}_{1}^{*}\right)=-\frac{1}{C_{2}}\left[k e^{\alpha t}\left(q_{1} q_{4} r_{1}-\bar{q}_{1} \bar{q}_{4} \bar{r}_{1}\right)-k e^{\alpha t}\left(q_{1} q_{4} r_{2}-\bar{q}_{1} \bar{q}_{4} \bar{r}_{2}\right)+\frac{p}{2}\left(q_{2} r_{3}-\bar{q}_{2} \bar{r}_{3}\right)\right],
$$

and

$$
\left(n_{r}^{*}-\bar{n}_{r}^{*}\right)=\frac{p e^{\alpha t}}{2 C_{3}}\left(q_{2}-\bar{q}_{2}\right)
$$

Assume that $r_{1}, r_{2}, r_{3}$, and $r_{4}$ are bounded by $N_{1}, N_{2}, N_{3}$, and $N_{4}$, respectively. Since $e^{-\alpha t} \leq 1$ for $t \geq 0$, then $q_{1}(t) \leq e^{-\alpha t} H_{s}(t) \leq H_{M}$, $q_{2}(t) \leq e^{-\alpha t} H_{i}(t) \leq H_{M}, q_{3}(t) \leq e^{-\alpha t} V(t) \leq V_{M} \quad y q_{4}(t) \leq e^{-\alpha t} T(t) \leq T_{M}$.

Finally, we notice that $e^{m t} \leq e^{m t_{f}}$, for all $t \in\left[0, t_{f}\right]$.

Taking differences $\left(\dot{q}_{i}-\dot{\bar{q}}_{i}\right),\left(\dot{r}_{i}-\dot{\bar{r}}_{i}\right), i=1, \ldots, 4$, multiply them by $\left(q_{i}-\bar{q}_{i}\right),\left(r_{i}-\bar{r}_{i}\right)$, respectively, and integrating from 0 to $t_{f}$, we obtain eight integral equations. The right hand sides of this equations are estimated using the bound of the optimality system, and Cauchy inequality in order to express the linear terms as sum of quadratic terms. After some algebraic manipulations and many calculations, we obtain expressions like the following:

$$
\frac{1}{2}\left[q_{1}-\bar{q}_{1}\right]^{2}\left(t_{f}\right)+\alpha \int_{0}^{t_{f}}\left(q_{1}-\bar{q}_{1}\right)^{2} d t \leq e^{\alpha t_{f}} K_{1} \int_{0}^{t_{f}}\left(q_{1}-\bar{q}_{1}\right)^{2} d t+e^{\alpha t_{f}} K_{2} \int_{0}^{t_{f}}\left(q_{3}-\bar{q}_{3}\right)^{2} d t,
$$


where

$$
\begin{aligned}
& K_{1}=e^{\alpha t_{f}} \\
& {\left[V_{M}+\frac{H_{M}}{2}+\frac{k^{2} H_{M} T_{M} e^{\alpha t_{f}}\left(N_{1}+N_{2}\right)}{C_{2}}\left(2 V_{M}+H_{M}\right)+\frac{k p H_{M} N_{3}}{C_{2}}\left(1+H_{M}\right)\right],}
\end{aligned}
$$

and

$$
K_{2}=e^{\alpha t_{f}}\left[\frac{H_{M}}{2}+\frac{k^{2} H_{M}^{2} T_{M} e^{\alpha t_{f}}\left(N_{1}+N_{2}\right)}{C_{2}}+\frac{k p H_{M}^{2} N_{3}}{C_{2}}\right] .
$$

Combining the estimates of the eight integral equations, we obtain

$$
\begin{aligned}
& \frac{1}{2}\left[q_{1}-\bar{q}_{1}\right]^{2}\left(t_{f}\right)+\frac{1}{2}\left[q_{2}-\bar{q}_{2}\right]^{2}\left(t_{f}\right)+\frac{1}{2}\left[q_{3}-\bar{q}_{3}\right]^{2}\left(t_{f}\right)+\frac{1}{2}\left[q_{4}-\bar{q}_{4}\right]^{2}\left(t_{f}\right) \\
& \quad+\frac{1}{2}\left[r_{1}-\bar{r}_{1}\right]^{2}(0)+\frac{1}{2}\left[r_{2}-\bar{r}_{2}\right]^{2}(0)+\frac{1}{2}\left[r_{3}-\bar{r}_{3}\right]^{2}(0)+\frac{1}{2}\left[r_{4}-\bar{r}_{4}\right]^{2}(0) \\
& \quad+\alpha \int_{0}^{t_{f}}\left[\left(q_{1}-\bar{q}_{1}\right)^{2}+\left(q_{2}-\bar{q}_{2}\right)^{2}+\left(q_{3}-\bar{q}_{3}\right)^{2}+\left(q_{4}-\bar{q}_{4}\right)^{2}\right. \\
& \left.\quad+\left(r_{1}-\bar{r}_{1}\right)^{2}+\left(r_{2}-\bar{r}_{2}\right)^{2}+\left(r_{3}-\bar{r}_{3}\right)^{2}+\left(r_{4}-\bar{r}_{4}\right)^{2}\right] d t \\
& \leq\left(\widetilde{K}_{1} e^{m t_{f}}+\widetilde{K}_{2}\right) \int_{0}^{t_{f}}\left[\left(q_{1}-\bar{q}_{1}\right)^{2}+\left(q_{2}-\bar{q}_{2}\right)^{2}+\left(q_{3}-\bar{q}_{3}\right)^{2}+\left(q_{4}-\bar{q}_{4}\right)^{2}\right. \\
& \left.\quad+\left(r_{1}-\bar{r}_{1}\right)^{2}+\left(r_{2}-\bar{r}_{2}\right)^{2}+\left(r_{3}-\bar{r}_{3}\right)^{2}+\left(r_{4}-\bar{r}_{4}\right)^{2}\right] d t,
\end{aligned}
$$

where $\widetilde{K}_{1}$ and $\widetilde{K}_{2}$ depend on the parameters of the state variables and upper bounds.

The above expression implies

$$
\begin{gathered}
\left(\alpha-\widetilde{K}_{1} e^{m t_{f}}-\widetilde{K}_{2}\right) \int_{0}^{t_{f}}\left[\left(q_{1}-\bar{q}_{1}\right)^{2}+\left(q_{2}-\bar{q}_{2}\right)^{2}+\left(q_{3}-\bar{q}_{3}\right)^{2}+\left(q_{4}-\bar{q}_{4}\right)^{2}\right. \\
\left.+\left(r_{1}-\bar{r}_{1}\right)^{2}+\left(r_{2}-\bar{r}_{2}\right)^{2}+\left(r_{3}-\bar{r}_{3}\right)^{2}+\left(r_{4}-\bar{r}_{4}\right)^{2}\right] d t \leq 0 .
\end{gathered}
$$


We now choose $\alpha$ and $t_{f}$ such that

$$
\ln \left[\frac{\left(\alpha-\widetilde{K}_{2}\right)}{\widetilde{K}_{1}}\right]>m t_{f},
$$

For this $\alpha$ and $t_{f}$, we have

$$
\alpha-\widetilde{K}_{1} e^{m t_{f}}-\widetilde{K}_{2}>0
$$

and we conclude that $q_{1}=\bar{q}_{1}, q_{2}=\bar{q}_{2}, q_{3}=\bar{q}_{3}, q_{4}=\bar{q}_{4}, r_{1}=\bar{r}_{1}, r_{2}=\bar{r}_{2}$, $r_{3}=\bar{r}_{3}$ and $r_{4}=\bar{r}_{4}$, in consequence $n_{1}^{*}=\bar{n}_{1}^{*}$, and $n_{r}^{*}=\bar{n}_{r}^{*}$. Thus, the Theorem 5 is proved. 